\title{
Transformation of Singular Light Beams at Nondegenerated Four-Wave Mixing in Dye Solutions
}

\author{
D. V. Gorbach, O. G. Romanov, and A. L. Tolstik \\ Belarusian State University, Minsk, Belarus \\ e-mail:Romanov@bsu.by
}

\begin{abstract}
An analysis of a frequency transformation of singular light beams by the dynamic hologram at a frequency nondegenerated four-wave mixing in dye solutions has been carried out. The transformation of optical vortices of the first and second order from the infrared spectrum to the visible one has been demonstrated experimentally with simultaneous inversion of the topological charge.
\end{abstract}

DOI: $10.3103 / \mathrm{S} 1062873809120211$

\section{INTRODUCTION}

The permanent interest taken recently in the singular optics accounts for the wide opportunities of using light beams with a spiral dislocation of the wavefront for optically capturing and manipulating microparticles, creating wave-guide structures of a specified shape, information transfer from one group of beams to another, conducting practical calculations, etc. For the transformation of a topological structure of the optical vortices, a second harmonic generation [4] and three- or four-wave mixing $[5,6]$ are used. Thus, for instance, at a four-wave mixing that was degenerated by the frequency in the colloidal medium, a phase conjugate of the vortex signal beam was demonstrated [7]. At a nondegenerated four-wave mixing in the vapors of the rubidium atoms, the subtraction of topological charges of two optical vortice was performed [8]. New opportunities open with the transformations of optical vortice which have several spectral components. The nonlinear dynamics of a bicolored optical vortice in the process of a second harmonic generating in lithium niobate crystals was examined in [9]. The formation of space-coherent single- and doublecharged polychromatic vortex solitons was shown in [10]. At the same time, for transforming the coherent images in real time, the methods of dynamic holography were developed [11, 12].

The main purpose of this paper is to show the opportunities for using the dynamic holographic methods of processing the optical images for the frequency transformation of the singular light beams. A theoretical analysis and numerical modeling of the phase conjugate processes of optical vortice in the scheme of a nondegenerated four-wave mixing were carried out, and the transformation of singular beams from the infrared spectrum into a visible one was experimentally demonstrated.

\section{THEORETICAL MODEL}

We performed a theoretical analysis of a four-wave mixing that was nondegenerated on the frequency by the example of transmitting dynamic lattices being recorded in the resonance medium alongside the propagating signal $E_{S}=A_{S} \exp \left[i\left(\mathbf{k}_{S} \cdot \mathbf{r}-\omega t+\varphi_{S}\right)\right]$ and background $E_{1}=A_{1} \exp \left[i\left(\mathbf{k}_{1} \cdot \mathbf{r}-\omega t+\varphi_{1}\right)\right]$ waves. The dynamic hologram is read using the counterpropagating wave $E_{2}=A_{2} \exp \left[i\left(\mathbf{k}_{2} \cdot \mathbf{r}-2 \omega t+\varphi_{2}\right)\right]$ at the doubled frequency $2 \omega$. During the process of mixing in the medium with resonance nonlinearity, a cubic polarizability $P=\chi^{(3)} E_{1} E_{2} E_{S}^{*}$ is induced which is responsible for the formation of a diffracted wave $E_{D}=A_{D} \exp \left[i\left(\mathbf{k}_{D} \cdot \mathbf{r}-2 \omega t+\varphi_{D}\right)\right]$. The efficient generating of the diffracted wave occurs in the direction determined by the condition of the phase synchronism $\mathbf{k}_{D}=\mathbf{k}_{1}+\mathbf{k}_{2}-\mathbf{k}_{S}$, which is equivalent to fulfilling the Bragg diffraction condition.

The system of the abridged wave equation, which describes the diffracted wave formation $E_{D}$ in the process of a nondegenerated four-wave mixing, may be written as follows:

$$
\begin{aligned}
& \left(\frac{\partial}{\partial z} \mp \gamma_{1, S} \frac{\partial}{\partial x}+\frac{\Delta_{\perp}}{2 i k_{1, S}}\right) E_{1, S} \\
= & \frac{i 2 \pi \omega}{c n_{0}}\left[\chi_{0}(\omega) E_{1, S}+\chi_{ \pm 1}(\omega) E_{S, 1}\right], \\
& \left(\frac{\partial}{\partial z} \pm \gamma_{2, D} \frac{\partial}{\partial x}+\frac{\Delta_{\perp}}{2 i k_{2, D}}\right) E_{2, D} \\
= & -\frac{i 4 \pi \omega}{c n_{0}}\left[\chi_{0}(2 \omega) E_{2, D}+\chi_{\mp 1}(2 \omega) E_{D, 2}\right],
\end{aligned}
$$

where $\gamma_{1}=\gamma_{S}$ and $\gamma_{2}=\gamma_{D}$ are the angles between the corresponding wave vectors $\left(\mathbf{k}_{1}, \mathbf{k}_{2}, \mathbf{k}_{S}, \mathbf{k}_{D}\right)$ and the axis $z, n_{0}$ is a nonresonance constituent of the medium refraction index (when a dye is used, it is determined 
by the solvent refraction index); $\Delta_{\perp}=\partial^{2} / \partial x^{2}+\partial^{2} / \partial y^{2}$ is a transverse Laplacian, responsible for the diffraction of spatially confined light beams; and $\chi_{0}$ and $\chi_{ \pm 1}$ are the components of expansion of the nonlinear medium susceptibility of $\chi_{n l}$ in the Fourier series: $\left.\chi_{N}=\frac{1}{2 \pi} \int_{-\pi}^{\pi} \chi_{n l} \exp \left[-i N\left(\left(\mathbf{k}_{1}-\mathbf{k}_{S}\right) \mathbf{r}\right)\right)\right] d\left(\left(\mathbf{k}_{1}-\mathbf{k}_{S}\right) \mathbf{r}\right), N=$ $0, \pm 1$. In this case, the mixing of the signal and reference waves is determined by the nonlinear susceptibility at the frequency $\omega$, while for both the reading and diffraction waves the susceptibility is relevant at a frequency of $2 \omega$.

A theoretical analysis has been carried out in the approximation of a two-state model of the medium considering both the resonance and the thermal mechanisms of nonlinearity, which are typical of the dye solutions. It is supposed that the medium absorbs radiation at the frequency of $\omega$ and it is transparent at the doubled frequency of $2 \omega$. In the given approximation, the nonlinear susceptibility can be represented as [13]

$$
\begin{gathered}
\chi_{n l}(\omega)=\frac{n_{0} \kappa_{0}}{2 \pi}\left(\frac{\hat{\Theta}_{12}}{B_{12}}-\frac{\hat{\alpha} I_{\Sigma}}{1+\alpha I_{\Sigma}}\right), \\
\chi_{n l}(2 \omega)=\frac{n_{0} \kappa_{0}}{2 \pi} \frac{a_{T} I_{\Sigma}}{1+\alpha I_{\Sigma}},
\end{gathered}
$$

where $\quad \hat{\alpha}=a+i \alpha=\left(\hat{\Theta}_{12}+\hat{\Theta}_{21}\right) / v P_{21}-a_{T}, \quad a_{T}=$ $\sigma_{T}\left(1-\mu_{21}\right)$. Parameter $\alpha=\left(B_{12}+B_{21}\right) / v P_{21}$ defines the saturation intensity of the resonance transition $S_{0}-S_{1}$ $\left(I_{\mathrm{SAT}}=\alpha^{-1}\right)$.

The intensity of the waves' interferential field $E_{1}$ and $E_{S}$ is found to be determined as $I_{\Sigma}=I_{1}+I_{S}+2 \sqrt{I_{1} I_{S}} \cos \left[\left(\mathbf{k}_{1}-\mathbf{k}_{S}\right) \mathbf{r}+\varphi_{1}-\varphi_{S}\right]$. In the expressions for the coefficients $\hat{\Theta}_{k l}(\omega)=$ $\Theta_{k l}(\omega)+i B_{k l}(\omega)$, the parameters $\Theta_{k l}(\omega)$ are connected by the Kramers-Kronig relations, with the Einstein coefficients for the forced transitions $B_{k l}(\omega)$ in the spectral channel $S_{0}-S_{1}$. The rest of the parameters may be defined in the following way: $v$ is a light velocity in the medium, $\kappa_{0}$ is an initial coefficient of extinction, $P_{21}$ is an integrated probability of spontaneous and nonradiative transitions, $\sigma_{T}=2 \omega(d n / d T) \tau / c C_{\rho}, \tau$ is the mixing duration, $C_{\rho}$ is a thermal capacity of the volume unit, $d n / d T$ is a thermo-optic coefficient, and $\mu_{21}$ is a quantum yield of luminescence in the channel $S_{0}-S_{1}$.

Using the Fourier expansion of nonlinear susceptibilities of $\chi_{n l}(\omega)$ and $\chi_{n l}(2 \omega)$, the equation system (1), (2) together with (3), (4) may be presented as

$$
\begin{gathered}
\left(\frac{\partial}{\partial z} \mp \gamma_{1, S} \frac{\partial}{\partial x}+\frac{\Delta_{\perp}}{2 i k_{1, S}}\right) E_{1, S}=i \frac{k_{0}}{2} f_{1, S} E_{1, S}, \\
\left(\frac{\partial}{\partial z} \pm \gamma_{2, D} \frac{\partial}{\partial x}+\frac{\Delta_{\perp}}{2 i k_{2, D}}\right) E_{2, D}=-i k_{0}\left[\psi E_{2, D}+\phi E_{D, 2}\right],
\end{gathered}
$$

where $k_{0}=2 \omega \kappa_{0} / c$ is the initial absorption coefficient

$$
\begin{gathered}
f_{1, S}=\frac{\hat{\Theta}_{12}}{B_{12}}-\frac{a}{\alpha}+\frac{\hat{\alpha}}{\alpha A}-\frac{2 \alpha I_{S, 1}}{A\left(1+\alpha\left(I_{1}+I_{S}\right)+A\right)}, \\
\psi=\frac{a_{T}}{\alpha}\left(1-\frac{1}{A}\right), \\
\phi=\frac{2 a_{T} \sqrt{I_{1} I_{S}}}{A\left(1+\alpha\left(I_{1}+I_{S}\right)+A\right)} \exp \left[ \pm i\left(\varphi_{1}-\varphi_{S}\right)\right]
\end{gathered}
$$

where $A=\left[1+2 \alpha\left(I_{1}+I_{S}\right)+\alpha^{2}\left(I_{1}-I_{S}\right)\right]^{1 / 2}$.

During numerical modeling it was assumed that the signal light beam contains the spiral phase dislocation of the topological charge $m$ : $E_{S}(z=0, r, \varphi)=E_{S 0}\left[\left(r-r_{S}\right) / r_{0 S}\right]^{|m|}$ $\exp \left[-\left(r-r_{S}\right)^{2} / 2 r_{0 S}^{2}+i m \varphi\right]$. Beams with a plane wavefront $E_{1}(z=0, r, \varphi)=E_{10} \exp \left[-\left(r-r_{1}\right)^{2} / 2 r_{01}^{2}\right]$, $E_{2}(z=L, r, \varphi)=E_{20} \exp \left[-\left(r-r_{2}\right)^{2} / 2 r_{02}^{2}\right]$ and a halfwidth three times as much than that of the signal one $\left(r_{01}=r_{02}=3 r_{0 S}\right)$ were used as the background $E_{1}$ and reading $E_{2}$ to efficiently record the dynamic lattices within the region of the beams' overlapping in the medium volume. The light beams recording the hologram were found to be crossed in the nonlinear layer at an angle of $2 \gamma_{1, S}=40 \mathrm{mrad}$, with the initial distance between their centers at the boundary $z=0$ being $r_{1}-r_{2}=r_{0 S}$. The half-width of the signal beam was supposed to be $r_{0 S}=1 \mathrm{~mm}$, the initial absorption coefficient of the nonlinear medium was $k_{0}=1 \mathrm{~cm}^{-1}$, the length of the nonlinear layer was $L=1 \mathrm{~cm}$, the background beam peak intensity was $\alpha I_{0}=1$, the intensity ratio was $I_{0 S} / I_{0}=0.1$, the radiation wavelength of $\lambda=$ $1 \mu \mathrm{m}$ coincided with the center of the absorption band of the resonance transition of the nonlinear medium, the refraction index was $n_{0}=1.36$, the thermo-optic coefficient was $\partial n / \partial T=-4 \times 10^{-4} \mathrm{~K}^{-1}$, the thermal capacity of the volume unit was $C_{\rho}=2 \mathrm{~J} \mathrm{~K}^{-1} \mathrm{~cm}^{-3}$, the pulse duration was $\tau=20 \mathrm{~ns}$, and the quantum yield of luminescence was $\mu_{21}=0.01$.

Since the regarded geometry of mixing of the light beams suggests the solution of the boundary problem while specifying the boundary conditions at various boundaries of a nonlinear medium (the fields $E_{1}$ and $E_{S}$ are determined at the boundary $z=0$, and the field of the reading wave $E_{2}$ is directed to the boundary $z=L$ ), the numerical modeling was performed in two stages: first, the propagation of the signal and reference 

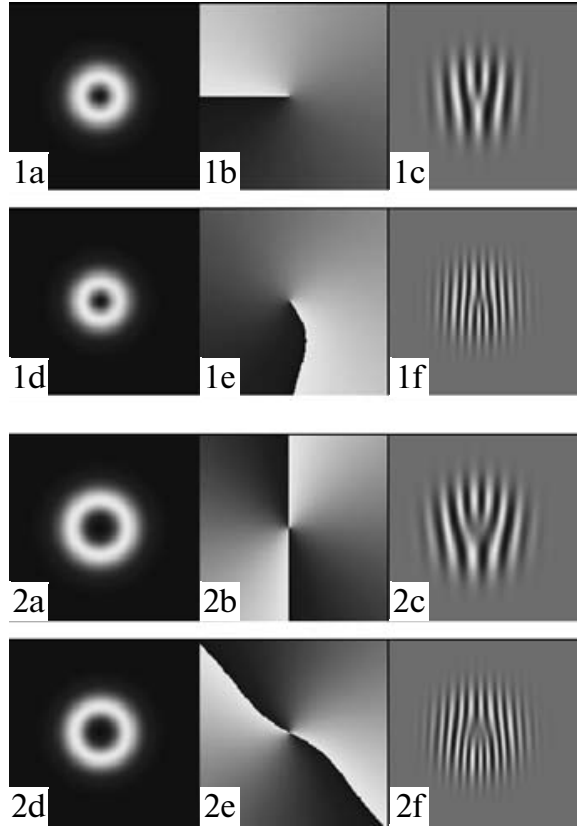

Fig. 1. Spatial propagations of the intensity $(a, d)$, phase $(b, e)$ and the interference patterns $(\mathrm{d}, \mathrm{f})$ for the signal $(\mathrm{a}$, $\mathrm{b}, \mathrm{c})$ and diffracted $(\mathrm{d}, \mathrm{e}, \mathrm{f})$ light beams with a topological charge $m=1$ (1a, 1b, 1c, 1d, 1e, $1 \mathrm{f})$ and $m=2$ (2a, 2b, 2c, $2 \mathrm{~d}, 2 \mathrm{e}$, and $2 \mathrm{f}$ ).

waves in the nonlinear medium volume was calculated (the direct solution of equation (5)); then a complete system of equations (5), (6) was solved in the opposite direction from the boundary $z=L$ to $z=0$, thus finding the spatial propagation of the reading and diffracted beams.

The results of a numerical analysis of the reading wave $E_{2}$ diffraction at the phase lattice, recorded by the Gaussian background beam $E_{1}$ and a singular signal beam $E_{S}$ with a topologic charge $m=1$ or $m=2$, are presented in Fig. 1. The ring distributions of the intensity (Figs. 1d, 2d) and the phase structures twisted in the cross section of the beam (Figs. 1e, 2e), which are typical of singular beams, are observed to form. In the interference patterns of signal and diffracted beams with plane reference waves, it is seen that the characteristic interferential forks have opposite directions (Figs. 1c, 1f; Figs. 2c, 2f). This implies that in the process of the four-wave mixing a diffracted wave $E_{D}$ is formed with the wavefront comprising the spiral dislocation of the opposite sign with respect to the signal wave $(m=-1$ or $m=-2)$. Here, as is seen from Figs. 1e and 2e, a phase shift and minor phase distortions for the diffracted beam occur, which are caused by the diffraction at the phase dynamic lattices under the conditions of the efficient power interchange. The double decrease of a period of the interferential picture for the diffracted wave versus the signal one is, perhaps, correlated with a switch to the doubled frequency during the dynamic hologram regeneration.

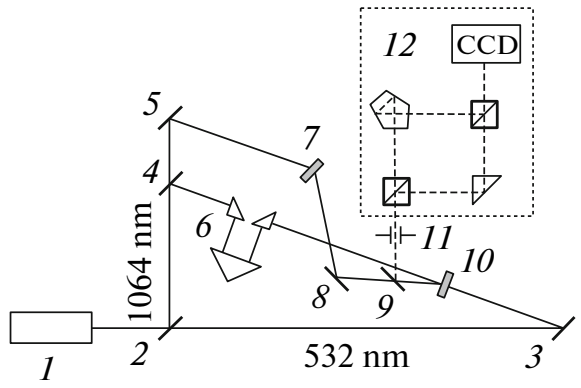

Fig. 2. Scheme of the experimental setup for implementing the frequency-nondegenerated four-wave mixing of the Gaussian and singular light beams and determining the topological charge value.

Note that the change of the topologic charge sign during the four-wave mixing follows from Eq. (6) as well, with the application of expression (9) for the coefficient of a parametric relation of the waves $\phi$. For the plane background and reading waves $\left(\varphi_{1}+\varphi_{S}=\right.$ const $)$, the phase of the diffracted wave is opposite the phase of the signal wave $\left(\varphi_{D}=-\varphi_{S}\right)$, which determines the inverting of the topologic charge of the diffracted beam with respect to the signal one.

\section{EXPERIMENTAL RESULTS}

The transformation of a topological structure of the singular light beams with their simultaneous frequency transformation has been experimentally performed in the ethanol solution of polymethine dye $3274 \mathrm{U}$ with a record of transmitting-type dynamic holograms in the the scheme of the co-propagating reference light beam and signal singular beam.

The scheme of the experimental setup is presented in Fig. 2. The experiments have been carried out with application of a yttrium aluminum garnet laser (1) (the radiation wavelength is $1064 \mathrm{~nm}$, the divergence of the light beam is $\theta_{0.5} \leq 2 \mathrm{mrad}$, and the pulse duration is $20 \mathrm{~ns})$. The dynamic holograms were recorded at the first harmonic of the laser radiation (1), corresponding to the maximum of the absorption band of the polymethine dye $3274 \mathrm{U}$ in ethanol. The dynamic holograms were restored by the second harmonic of radiation of the same laser at a wavelength of $532 \mathrm{~nm}$, which was not actually absorbed by the dye solution. Signal $E_{S}$ and background $E_{1}$ waves were being formed by the beam splitter $(2)$ and mirrors $(4,5)$. Delay line $(6)$ was used so that the optical length of the path of the signal and reference waves are equal. In order to obtain a signal light beam with a spiral dislocation of the wavefront, special computer-synthesized holograms (7) were used that were recorded in the layers of polymethylmethacrylate containing phenanthrenequinone with a diffraction efficiency of about $10 \%$. The singular beam was directed into the dye solution containing cell $(10)$ at a small angle to the reference wave provided by reflector $(8)$. The intensities of the back- 


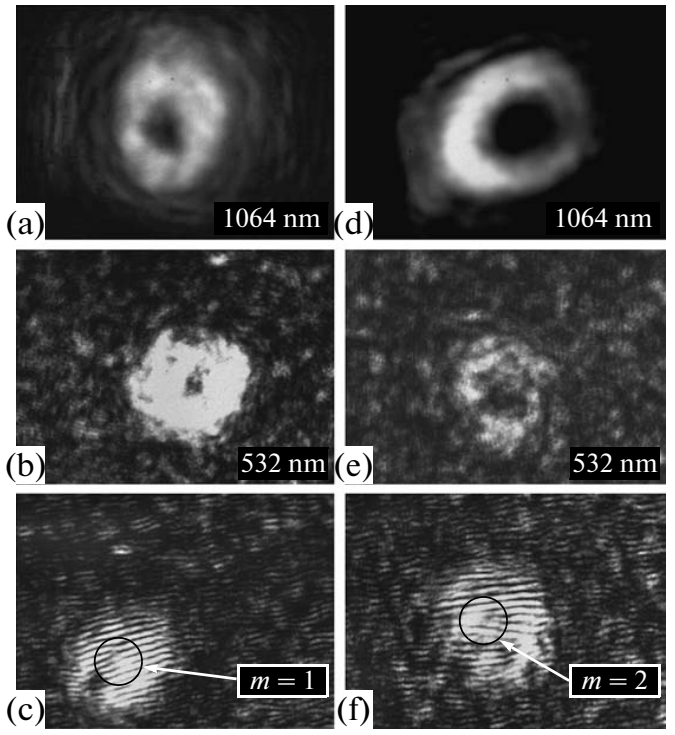

Fig. 3. The measured spatial profiles of the signal (a, d) and the diffracted $(b, e)$ light beams and interference patterns for the diffracted beams (c, f) with the topological charge $\mathrm{m}=1(\mathrm{a}, \mathrm{b}, \mathrm{c})$ and $m=2(\mathrm{~d}, \mathrm{e}, \mathrm{f})$.

ground and signal waves equaled $I_{1}=1 \mathrm{MW} \mathrm{cm}^{-2}$ and $I_{S}=0.1 \mathrm{MW} \mathrm{cm}^{-2}$.

The reading wave $E_{2}$ at the doubled frequency of $2 \omega$ was directed into the cell containing the dye solution at a small angle and against the reference wave $E_{1}$ by a reflector (3). The direction of the reading wave propagation was determined by the condition of the phase-matching for the frequency nondegenerated four-wave mixing $\left(\mathbf{k}_{1}-\mathbf{k}_{S}=\mathbf{k}_{D}-\mathbf{k}_{2}\right)$. The small angle $\left(2 \gamma_{1, S} \approx 30 \mathrm{mrad}\right)$ between the propagation directions of the background and signal waves and the cross dimensions of the beams $\left(r_{0}=250 \mu \mathrm{m}\right.$ and $r_{0 S}=75-$ $150 \mu \mathrm{m})$ provided the efficient spatial overlapping of all the interacting light beams in the dye solution cell. To separate the diffracted beam, a senai-transparent mirror (9) and diaphragm (11) were used. The spatial profiles of the light beams' intensity were registered by a CCDcamera located at the output of the Mach-Zender interferometer 12, which enables one to obtain interference patterns for the signal and diffracted light beams and identify their topological structure.

The results of the experimental investigations of the frequency nondegenerated four-wave mixing in the ethanol solution of polymethine dye $3274 \mathrm{U}$ are displayed in Fig. 3. As can be seen, due to the diffraction of the reading wave $E_{2}$ at the phase dynamic hologram recorded by the reference wave $E_{1}$ and signal wave $E_{S}$, which contain the spiral dislocation of the wavefront with a topological charge of $m=1$ (Fig. 3a), the diffracted wave $E_{D}$ is formed with a similar topological charge, but at twice the frequency (Fig. 3b). The interferential picture obtained by the Mach-Zender inter- ferometer is characterized by the presence of a single forked-shape dislocation typical for singular beams with a single topological charge $m=1$ (Fig. 3c).

Similarly, if a beam with a doubled topological charge $m=2$ is used as a signal light beam, a singular diffracted beam (also containing a doubled topological charge, but at a doubled optical frequency) is formed as a result of the nondegenerated four-wave mixing.

\section{CONCLUSIONS}

The presented theoretical analysis, along with the numerical modeling of the system of the wave equations describing the process of a frequency-nondegenerated four-wave mixing in the dye solutions, provided for the opportunity to achieve the inversion of a topological charge sign of the singular light beams with the simultaneous frequency transformation of the signal. The offered method for the transformation of a topological charge of the singular beams on the basis of the scheme of a nondegenerated four-wave mixing in the ethanol solution of polymethine dye $3274 \mathrm{U}$ was used to accomplish the frequency transformation of the optical vortice of the first and second order from the IR-spectrum to the visible one.

\section{REFERENCES}

1. Gahagan, K.T. and Swartzlander, G.A., Opt. Lett., 1996, vol. 21, p. 827.

2. Kivshar', Yu.S. and Agraval, G.P., Opticheskie solitony. Ot volokonnykh svetovodov $k$ fotonnym kristallam (Optical Solitons: from Fibers to Photonic Crystals), Moscow: Fizmatlit, 2005; San Diego: Academic Press, 2003.

3. Roychowdhury, S., Jaiswal, V.K., and Singh, R.P., Opt. Commun., 2004, vol. 236, p. 419.

4. Courtial, J., Dholakia, K., Allen, L., and Padgett, M.J., Phys. Rev. A, 1997, vol. 56, p. 4193.

5. Berzanskis, A., Matijosius, A., Piskarskas, A., et al., Opt. Commun, 1997, vol. 140, p. 273.

6. Pyragaite, V., Regelskis, K., Smilgevicius, V., and Stabinis, A., Opt. Commun, 2001, vol. 198, p. 459.

7. Lopez-Mariscal, C., Gutierrez-Vega, J.C., McGloin, D., and Dholakia, K., Opt. Express, 2007, vol. 15, p. 6330.

8. Jiang, W., Chen, Q., Zhang, Y., and Guo, G.-C., Phys. Rev. A, 2006, vol. 74, p. 043811.

9. Dreischuh, A., Neshev, D.N., Kolev, V.Z., et al., Opt. Express, 2008, vol. 16, p. 5406.

10. Neshev, D.N., Dreischuh, A., Shvedov, V., et al., Opt. Lett., 2008, vol. 33, p. 1851.

11. Acioli, L.H., Gomes, A.S.L., Rios Leite, J.R., and de Araujo, C.B., Appl. Phys. Lett., 1989, vol. 54, p. 1956.

12. Ormachea, O., Romanov, O.G., Tolstik, A.L., et al., Opt. Express, 2006, vol. 14, p. 8298.

13. Tolstik, A.L., Mnogovolnovye vzaimodeistviya $v$ rastvorakh slozhnykh organicheskikh soedinenii (Multiwave Interactions in Solutions of Complex Organic Compounds), Minsk: BGU, 2002. 